Matjaž Birk*

Universität Maribor
UDK 398.2(497.434=112.2)

DOI: 10.4312/linguistica.60.2.335-346

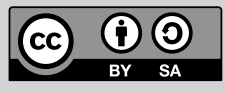

\title{
VOLKSSAGEN DER GOTTSCHEER: ASPEKTE DES RAUMES
}

\section{HISTORISCHE KONTEXTE UND TEXTE ${ }^{1}$}

[...] Sie lebte im Ohr des Volks, auf den Lippen und der Harfe lebendiger Sänger: sie sang Geschichte, Begebenheit, Geheimniß, Wunder und Zeichen: sie war die Blume der Eigenheit eines Volks, seiner Sprache und seines Landes, seiner Geschäfte und Vorurtheile, seiner Leidenschaften und Anmassungen, seiner Musik und Seele. [...] (Herder 1889: 62f.)

schrieb Herder in der Vorrede zu seiner 1778 und 1779 erschienen Sammlung Volkslieder, die in Annalen der Weltliteratur unter ihrer späteren Titelbezeichnung Stimmen der Völker in Liedern bekannt wurde. Der Autor gilt als Begründer der Theorie und Geschichte der Volksdichtung, mit seinen Volksliedern lieferte er entscheidende Impulse für Sammlung, Erforschung und Transfer von Volksdichtung im deutschen Sprachraum wie in jenen an ihn gebundenen Kommunikationsräumen in Mittel- und Südosteuropa. So erfasste die Herdersche Verve auch die auf dem Gebiet des heutigen Slowenien lebende ethnische Gemeinschaften, unter ihnen auch die Gottscheer. Neben Adolf Hauffen, dem Begründer der wissenschaftlichen Erfassung der Sprache, Literatur und Kultur der Gottscheer, der 1895 beim Universitätsverlag in Graz die bahnbrechende Monographie Die deutsche Sprachinsel Gottschee (Hauffen 1895) herausbrachte, nimmt in der Sammlung der Volkskultur der Gottscheer einen besonderen Stellenwert Wilhelm Tschinkel ein. Der aus einer vermögenden Lodenfabrikantenfamilie aus Lichtenbach (slow. Svetli Potok) stammende, in Marburg an der Lehreranstalt ausgebildete, in Rieg (slow. Kočevska Reka), Morobitz (slow. Borovec), Gottschee (slow. Kočevje) und ab 1924 in Rosegg (slow. Rožek) in Kärnten wirkende Lehrer und Schuldirektor erhielt für die Sammlung der literarischen Folkloristik seiner Landsleute wichtige Anregungen von seinem namhaften Onkel, Hans Tschinkel, dem Verfasser der 1908 in Halle erschienenen Grammatik der Gottscheer Mundart. Im Jahr 1932 erschien dank der finanziellen Unterstützung des wohlhabenden Vetters Alois Kraker, des Großkaufmanns aus Pettau, bei der Pavliček Buchdruckerei in Gottschee Wilhelm Tschinkels Lebenswerk - die den Titel Gottscheer Volkstum in Sitte, Brauch, Märchen, Sagen, Legenden und anderen volkstümlichen Überlieferungen führende Sammlung der Volksliteratur seiner

matjaz.birk@um.si

1 Der Beitrag entstand im Rahmen der von der slowenischen Forschungsagentur (ARRS) geförderten Programmgruppe Interkulturelle literaturwissenschaftliche Studien (P6-0265). 
Landsleute. ${ }^{2}$ Im Vorwort zur Sammlung bringt Tschinkel ihre Zielsetzung unmissverständlich zum Ausdruck - „diese Dinge vor unverdienter Vergessenheit zu bewahren und um zu beweisen, daß nicht bloß etwa nur die anderen Zweige des deutschen Volkes reich sind an volkstümlichen Überlieferungen, sondern daß auch bei uns in Gottschee ein reicher Schatz alten Volkstums verborgen liegt" - und grenzt sich von Opponenten in eigenen Reihen entschieden ab:

\begin{abstract}
So selbstverständlich diese Bemerkungen für denjenigen klingen, der sich mit diesen Fragen schon einmal beschäftigt hat, so notwendig scheinen sie mir für diejenigen, die da meinen, vieles von dem, was da gebracht wird, sei geeignet, unser Ländchen vor den Augen der Welt herabzusetzen. Diesen Leuten, die in solchen Dingen noch genau so urteilen, wie man vor hundert Jahren geurteilt hat, gelten vor allem diese einleitenden Worte. (Tschinkel 2004: 318)
\end{abstract}

In den Wirren der Zeit geriet Tschinkels Gottscheer Volkstum lange in Vergessenheit. Seit 2004 steht es als kritische Ausgabe im Original und in slowenischer Übersetzung der Leserschaft wieder zur Verfügung.

Die Sammlung enthält Märchen, Sagen, Legenden, Schwänke, Sprichwörter, Rätsel, Kinder- und Erwachsenenspiele, Auszählreime usw. Den weitaus größten Teil nehmen die Sagen ein. Die Gattungsbestimmung der Sagen (altnordisch ist Sagas ,Erzählung') ist ein Sammelbegriff für stark unterschiedliche Inhalte; geringer Wortschatz und Formelhaftigkeit gehören zu wichtigen Gattungsmerkmalen, wobei der gemeinsame Nenner „das Unerhörte, das Rätselhafte“ sei, das „,dem Menschen [...] in der Gestalt des Übernatürlichen und Unerklärlichen“ in der "das Übliche weit übersteigenden Tat" (Bausinger 1980: 188) entgegentritt. Tschinkels Sammlung enthält insgesamt 88 Sagen, wobei innerhalb einer Sage häufig mehrere Kurznarrative miteinander verkettet werden. Die Sagen, die in 13 Subgattungen unterteilt werden - in „Elbische Sagen“ bzw. Geistersagen, Gespenster-, Hexen-, Schatzgräber-, Teufels-, Schlangen-, Versteinerungs-, Gewässer-, Wurzeln-, Kirchen- und Kapellen-, Geschichts-, Heimkehr- und Inzestsagen -, kennzeichnet eine besondere Urwüchsigkeit stofflicher und thematischer Überlieferung, die als Produkt des inselhaften Charakters des Sprachgebiets ${ }^{3}$ zu betrachten sei.

\title{
2 THEORETISCHE ÜBERLEGUNGEN
}

Globale Enträumlichung - Globalisierung mit der Tendenz zur Ortlosigkeit - und postkolonialistische Entwicklungen erzeugten in der Postmoderne - nach der starken Betonung der Kategorie der Zeit in der Moderne - ein gesteigertes Interesse der Kulturwissenschaften für den Raum, was in der Analogie zu anderen Neufokussierungen mit dem Syntagma räumliche Wende bezeichnet wurde. Diese hatte Anerkennung von

2 Von W. Tschinkel ging des Weiteren auch die Anregung für den Gottscheer Kalender aus, den er am Anfang seines Erscheinens, in den Jahren 1921-1923, auch redigierte. Neben W. Tschinkel treten als Sammler der Gottscheer Volksliteratur auch einige andere namhafte Gottscheer auf, unter ihnen Josef Perz, der erste Volksliedsammler, und Wilhlems Onkel, Hans Johann Tschinkel.

3 Vgl. Bredhnich (1996), Krevs Birk (2019). 
Gleichzeitigkeit verschiedener Kulturen und kritisches Re-Mapping der bisher hegemonialen Zentren und marginalisierten Peripherien zur Folge. Der Raum erscheint als Analysekategorie und Konstruktionsprinzip sozialen Verhaltens, als Dimension der Materialität und Repräsentationsstrategie. Während Verzeitlichung auf Diachronie, Geschichte, Entwicklung und Fortschritt fokussiert, lässt Verräumlichung Synchronie, Materie, Stoff und Konstellationen in Erscheinung treten. In der medialen Repräsentation des Raumes gehen essentialistische und antiessentialistische Konzepte einher: Repräsentationen von Raum als Behälter von Traditionen - Raum als Nationalstaat oder gar als Heimat - werden verknüpft mit Repräsentationen des Raumes als dynamische Kategorie mit fließenden Übergängen, zahlreichen Grenzüberschreitungen und Überlappungen. Alle literarischen Beschreibungen enthalten Raumformen - von Kultur- bis Naturräumen, von Zimmer bis Nest -, daher ist Literatur Erzeugung bzw. Poetik von Raum. Unser Ziel war es, ausgehend von den durch Henri Lefebvre (Lefebvre 2006 (1974)) begründeten und durch den Sozialgeographen Edward Soja (Soja 2005) gedeuteten raumtheoretischen Konzepten, besonders des Drittraums-Konzepts ${ }^{4}$ als „Feld zur Einschreibung von Differenzen“" (Breger/Döring 1998: 2), die innere Raumdialektik in dem von Tschinkel herausgegebenen gottscheerischen Sagengut zu beleuchten. Henri Lefebvre versteht Räume als raumproduzierende Dialektiken und unterscheidet zwischen Dialektik innerhalb des Textes, der Dialektik außerhalb des Textes und einer dritten Dialektik, die aus der Verbindung der beiden zu einem übergreifenden kulturhistorischen Repräsentationsraum wird. ${ }^{5}$ Während die externe Dialektik die Beziehungen zwischen dem Schreiben, der Verbreitung und der Rezeption des Werkes (einschließlich transmedialer Anverwandlung) in räumlicher Hinsicht darstellt ${ }^{6}$, schließt die raumproduzierende Dialektik innerhalb des Narrativs (bzw. die innere Raumdialektik) literarische Raumpraxis, Raumrepräsentation und Repräsentationsraum ein. Auf diese Dialektik wollen wir in unserer analytischen Beschreibung fokussieren und die Frage nach Verknüpfung zwischen literarischer Raumrepräsentation und dem Repräsentationsraum der $\mathrm{Sage}^{7}$ anschneiden.

\section{ANALYSE DER INNEREN RAUMDIALEKTIK: RAUMPRAXIS, RAUMREPRÄSENTATION UND REPRÄSENTATIONSRAUM}

Literarische Raumrepräsentationen im geographischen Sinne handeln von Orten und können in der Schilderung von Räumen bestehen. Erzählte Räume dienen als Handlungsräume, die verschiedene Aspekte einschließen, unter ihnen Handlungen der

4 Der Begriff an sich ist eine Wortschöpfung des postkolonialen Theoretikers Homi K. Bhabha, der von E. Soja konzeptualisiert wurde.

5 Das Narrativ erscheint als perzipierter Raum und das Medium als repräsentierender Raum mit ihren jeweiligen Binnendialektiken.

6 In der externen Dialektik geht es um die Lokalisierung der in den Texten beschriebenen Schauplätze und um die Verbindung zu den Orten der Textproduktion und des Textvertriebs wie auch um den Raum der Herstellung und Verbreitung des Textes.

7 Die Gründungssage rührt von der Repräsentation des Lochs, in dem ein Ungeheuer haust (Tschinkel 2004: 419). 
Figuren, die Zusammenhänge innerhalb des erzählten Raums in den Blick nehmen. In den Sagen sind die Hauptträger der Handlungen Gottscheerinnen und Gottscheer sowie aus dem Gottscheerlande stammende überirdische Wesen. Die zur Welt der Menschen zugehörigen Figuren entstammen meist dem dritten Stand - es sind dies Bauern, Winzer, Wirte, Handwerker, Müller, Holzfäller, Mägde, Knechte, Dienstboten, Jäger, Förster, Gendarmen, Offiziere usw. Den zweitwichtigsten Stellenwert in der Handlungsstruktur nimmt der Klerus ein - Pfarrer und Geistliche aus der Gottschee, teilweise auch aus dem benachbarten Kroatien. An einigen wenigen Stellen treten auch die Repräsentanten des Adels vor. Die Identiät menschlicher Protagonisten wird vom Postulat des identifikatorischen Lesens gekennzeichnet. Diesem wird Rechnung getragen durch die Angabe realexistierender Orte und durch Zuweisungen von historischen (bzw. historisch anmutenden) Haus-, Personen- und Spitznamen, ${ }^{8}$ was zugleich auch als Gattungsspezifik des Repräsentationsraums der Sage erscheint. Unter den Figuren befinden sich auch zahlreiche geisterhafte und gespenstische Weiber- und Männergestalten sowie Tiere - weiße und wilde Frauen, wilde Männer, Bilchhmännchen (Siebenschläfermännchen), Waldmännchen, Kobolde, Teufel, Hexen, Lindwürmer, gespenstische Rosse und Riesenschlangen - sowie identitäre Hybride wie etwa die sich in Hexen verwandelnden Ehefrauen oder aus Schlangen hervorgegangenen Ehemänner usw. ${ }^{9}$ Die menschlichen Figuren treten mit dem Übernatürlichen in narrativ, szenisch und diskursiv entwickelten Kontakt meist außerhalb des Hauses, in der Natur, auf dem Feld, in dem Wald, auf einer Erhöhung und in der Untererde - an repräsentierten Orten, die geographisch durch den „wildromantischen Kulpatale“ (Tschinkel 2004: 407) eingegrenzt werden. Zum Kontakt mit dem Übernatürlichen kommt es aber auch in inneren Räumen, in Häusern und Wirtshäusern, Scheunen, Zimmern, Stuben, Kellern, Weinkellern, Dachböden, Rauchfängen, auf Treppen usw. Eine auf das Innere bezogene räumliche Kuriosität stellt die Studierstube dar, in der der Ortspfarrer nach Rat für trostlose, den Geistern ausgelieferte Pfarrmitglieder sucht (ebd.: 374). Unter den Naturräumen kommt in der Raumstruktur den Unterirdischen besonderer Stellenwert zu, was angesichts der geografischen Konfiguration des Gebietes nicht verwundert. Der Karst wird zu einem der zentralen, an der Erzeugung des Übernatürlichen beteiligten Raumfaktoren. Zu karsttypischen Räumen bzw. unterirdischen Handlungsorten gehören Löcher, auch solche, denen das karge Wasser entspringt, also Quellen und Brunnen, des Weiteren unterschiedliche Gruben, Höllen und Grotten, in denen Kobolde, Schlangen

8 „Bei Burschtaisch (Hausname) in Muckendorf [...](Tschinkel 2004: 401), „Ein gewisser Andreas Schneller aus Lichtenbach[...]“"(ebd.: 376), ,[...] ein gewisser Jakob Weber, der den Winter hindurch in Kärnten hausierte [...],“ (ebd.: 396), „Auch ein ehemaliger Besitzer von »Schimonsch « Hause in Morobitz, ein gewisser Andreas Michitsch [...]“(ebd.: 396), „Die Mutter des längst verstorbenen »Garibaldi« (Spitzname eines gewissen Andreas Jonke) in Kummerdorf [...]“ (ebd.: 373) usw.

9 Teilweise lehnt sich die Identitätskonstruktion der Figuren an im deutschen Mythen- und Sagenreservoir enthaltene Narrative, unter diesen auch über Gespensterritter und faustische Gestalten, denen räumlich- und kulturspezifische Identitätsmerkmale zuteil werden. So geht der Gottscheer zwecks materieller Versinnlichung der Existenz einen Pakt mit dem Bösen ein (ebd.: 400). 
und andere Schauderkreaturen hausen. Die Raumrepräsentationen weisen eindeutig Gegenwartsbezug auf, was besonders in zahlreichen Gespenster-, Hexen-, Schatzgräber-, Teufels-, Gründungs- und teilweise auch in Geschichtssagen zum Vorschein tritt:

Nahe an dem Fußsteige, der vom schön gelegenen Pfarrdorfe Ebental nach Tiefental führt, ist ein Loch, wie sich solche im Karste häufig finden. An kalten Morgen kann man einen dünnen Nebel daraus aufsteigen sehen, den aber die Leute für Rauch halten, denn sie behaupten, dieses Loch führe bis in die Hölle hinunter und sei gleichsam der „Schornstein der Hölle“. Einmal nun trieben sich übermutige Hirtenbuben in der Nähe des Loches herum und warfen Steine hinein. Plötzlich erschien der Teufel in Gestalt eines roten Hundes [..] (Tschinkel 2004: 407)

Der Einbruch des Übernatürlichen, sei es des Numinosen oder Magischen, leitet häufig einen schnellen Schauplatzwechsel ein, der von changierenden (Macht)verhältnissen zwischen dem Irdischen und Überirdischen bestimmt und mit unerwarteten Handlungswenden verknüpft wird, was an folgender Stelle aus der Sage über ein Gespensterpferd deutlich zu sehen ist:

Ein Knecht aus Ribnik ging eines Nachts angeheitert von Neuberg nach Hause. Als er unterhalb Muckendorf zu einer Buche kam, an der ein Heiligenbild hing, hörte er ein Geräusch im Gebüsch. Gleich darauf stand ein Schimmel mit feurigen Augen, so groß wie ,urhobzautlain“ (Sauerteigbrote), vor ihm und versperrte ihm den Weg. Er wollte das Pferd vertreiben, doch es schlug mit seinen Hinterbeinen so gewaltig aus, daß die Funken stoben. (ebd.: 380)

Die Raumpraxis ist im Unterschied zu Raumrepräsentation vorwiegend der Vergangenheit zugehörig, dennoch wird auch sie entscheidend von der Begegnung mit dem Übernatürlichen geprägt. Verortet zwischen der standesgemäßen Führung oder im Widerspruch mit Stand und Sitten stehenden Verhaltens- und Handlungsweisen, eingebettet in sich rasant verändernde Machtverhältnisse und -konstellationen, besteht die Raumpraxis aus einer Kette von sich wiederholenden Verhaltens- und Handelspraxen, die Herausforderung, Beschwörung, Betrug an dem Übernatürlichen wie auch dessen Bezwingung einschließen. Dabei oszillieren Protagonisten zwischen handlungsrelevanten Orten und im Netzwerk zwischen dem Irdischem und Überirdischen verorteten Figuren. In Entsprechung mit dem Repräsentationsraum der Sage steuert die Raumpraxis die finale Wendung zum Guten an, wie dies anhand des folgenden Narrativs aus der Kategorie der Schlangensagen deutlich zu sehen ist. Darin geht ein Mädchen die Ehe mit einem Schlangensohn ein. Dieser wird dank der Liebenswürdigkeit seiner nun Ehefrau erlöst, die er allerdings daraufhin verlässt, weil sie entgegen seinem Wunsch die vermeintlich glücksbringende Schlangenhaut aus dem Hause weggeschafft hat:

Sobald er heimkehrte und dieses vernahm, verließ er sie. Sie aber war guter Hoffnung und konnte nun nicht gebären sieben Jahre lang. Da zog sie aus nach ihrem Mann. Auf dem Wege begegnete ihr eine weiße Frau. Die sagte ihr: „Dein Mann 
lebt mit einer Zauberin in ihrem Schlosse, da nimm die Spielzeuge!" Sie nahm die Spielzeuge und gab zwei der Zauberin, damit sie mit ihrem Manne reden könne. Als sie aber zu ihm kam, schlief er noch von einem Zaubertrank; da gab sie das letzte Spielzeug hin. Nun vermied er den Zaubertrank, sprach mit dem Weibe und sie gebar einen Sohn. Die Zauberin ward vertrieben und sie lebten noch lange miteinander. (ebd.: 409)

Überall dort, wo Raumrepräsentationen, dem Repräsentationsraum der Sage entsprechend auf geringen Wortschatz beschränkt und von einem allwissenden Erzähler vermittelt werden, verschaffen sie Zutritt zu den für die Leser in der Realität schwerzugänglichen Naturräumen der Karstlandschaft:

In der Nähe von Reichenau liegt in wildromantischer Gegend eine Grube, die „Vaichtgruřbə" (Fichtengrube) genannt wird. Auf der einen Seite ist sie leicht zugänglich, auf der anderen türmen sich hohe Felsen auf. Am Grunde dieser Grube öffnet sich ein grottenähnlicher Eingang, doch kann man wegen des Steingerölls und des steil abfallenden Bodens nicht weit vordringen. [...] (ebd.: 406)

Handlungen erzeugen nicht nur Räume, sondern auch Stimmungen, was besonders deutlich in zahlreichen Geistersagen zu sehen ist. Dabei brauchen die Stimmungen nicht explizit im Detail ausgeführt zu werden, denn der Raum selbst expliziert sie:

In Handlern spann eine Frau am Luzienabend (13. Dezember) bis tief in die Nacht hinein. Da kam jemand und warf eine Menge leerer Spulen ins Zimmer, wobei eine Stimme sprach: „Diese Spulen mußt du heute alle noch anspinnen, sonst ergeht es dir schlecht!“- Die Frau erschrak, ging zur Nachbarin und fragte, was sie machen solle. Diese erteilte ihr den Rat, um jede Spule nur einen Faden zu wickeln. Als sie getan hatte, wie ihr geheißen war, kam dieser jemand wieder und wieder erscholl die Stimme: »Es ist dein Glück, daß du darauf verfallen bist, denn sonst wäre es dir schlecht ergangen.“(ebd.: 364)

Der Einbruch des Naturmagischen verleiht Vertrautheit und Geborgenheit spendenden (Natur)Räumen den Charakter der Fremdheit, der sich als Unergründbarkeit des Lebens niederschlägt: Angesichts der dystopischen Naturerscheinung erscheint die vom beispielhaften Arbeitswillen herrührende existentielle Stabilität der weiblichen Hauptprotagonistin Perchta bzw. Bertha (ebd.: 363) als brüchig und die Rätselhaftigkeit der Natur greift somit auch auf die menschliche Existenz über. Die Gottscheer begegnen der Unergründbarkeit der Natur und des Menschen als Natur- und Kulturwesen mit Demut und Hellhörigkeit.

Zur Verräumlichung der existentiellen Unergründbarkeit werden Kulturräume erweitert durch das räumliche Andere des Adelswohnsitzes, wie dies der Fall in einer sich im Schloss Friedrichstein abspielenden Schatzgräbersage (ebd.: 397) ist. Die Repräsentation der räumlichen Alterität des Schlosses schließt die Erzeugung eines zwischen 
dem Dies- und Jenseits liegenden Drittraumes ein, der im konkreten Fall räumliche Verortung in einem „hellerleuchtenden Saal“ mit „totenblaßen“ Männern und Frauen, unter denen „keiner [...] ein Wort“ (ebd.: 372) spricht, erfährt. Der zwischen Realem und Numinosem verortete Drittraum erscheint als zentrale räumliche Rekurrenz auch in vielen Sagen der Kirchen und Kapellen wie etwa in der Gründungssage über die Kirche auf dem Friedensberge (ebd.: 418f.).

Neben der Handlung kommt bei der Verräumlichung besondere Bedeutung auch magischen Gegenständen und Pflanzen zu. Unter ihnen befinden sich Knäule, Salben, Schißlinge (einjährige Haselruten), Kerzen, Kreuze, Heiligenbilde, Krampe, Sensen, Turmuhren, Besen sowie Päckchen, Ofengabel, Deckel, vereinzelt auch magische Pflanzen - so wird dank einer Pfefferstaude, die bei Gefahr aufblüht, die Türkengefahr abgewendet (ebd.: 426). Diese und die genannten Kulturprodukte werden zur Raumrepräsentation angewendet, indem sie in ihrer Eigenschaft des Magischen vertraute Räume in eine befremdende Perspektive rücken, in der Nähe und Vertrautheit verfremdet werden, wie dies der Fall in folgender Hexensage ist:

In Stockendorf lebte einst eine gefürchtete Hexe. Dies betrog ihren Mann jeden Abend, indem sie auf ihr Bett einen Besen legte, während sie zum Hexentanz auf den Berg Kleck fuhr. Ihr Mann schöpfte Verdacht und stellte sich eines Abends schlafend. Da sah er ganz deutlich, wie seine Frau eine Salbe nahm, sich damit bestrich und dabei die Worte sprach: „Hi trk, nindrt et uən! (Hi trk, nirgends an!) In demselben Augenblicke fuhr sie auch schon durch den Rauchfang davon. Da erhob sich der Mann aus dem Bette, nahm die geheimnisvolle Salbe und bestrich damit einen Baumklotz im Garten [...] Da begann sich der Klotz zu rühren [...] (ebd.: 387)

Auf die Destabilisierung der vertrauten Handlungspraxis folgt nach dem abgeschlossenen magischen Zwischenspiel nicht nur Konsolidierung von Raum, sondern auch Stabilisierung von Raumpraxis, die auf einer erweiterten sinn- und identitätsstiftenden Grundlage erscheint. So tritt das Unheimliche nicht als Gegenteil des Heimlichen zum Vorschein, sondern als eine im Hinblick auf den Einzelnen und das Kollektiv produktiv erscheinende Verschiebung der Vertrautheit am angestammten Ort. In einigen Sagen sorgen magische Gegenstände - in der erwähnten Sage über den Schlangensohn (ebd.: 408) ist es das wunderbare Spielzeug - für Verrückung des Heimlichen, wobei die Wendung zum Heimlichen am unheimlichen Ort am Ende von der Rückverschiebung des Heimlichen und Vertrauten am angestammten Ort überblendet wird.

Die Sagen sind Repräsentationsraum des vormodernen Denkens, das von generationenübergreifendem Volksaberglauben, innigem Glauben und einer Haltung gegenüber der Natur gekennzeichnet ist, die die Signatur der an Adalbert Stifter gemahnenden Naturphänomenologie trägt. Zugleich jedoch kommt in ihnen auch eine im Rationalismus begründete Modernität zum Vorschein. Die schlägt sich nieder im ironischen Unterlaufen des inszenierten Aberglaubens (,es gebe ja gar keine Hexen“, ebd.: 391), in der Kritik an Beispielen normierter, sich seicht anmutender Volksreligiosität (,so 
hatte sie eines Abends tausend Vaterunser heruntergeleiert, indem sie hierbei Fisolen zählte, um sich nicht zu irren“, ebd.: 395), an moralischer Doppelbödigkeit des kirchlichen Machtraumes - dass diesem vonseiten der Gottscheer mit Nulltoleranz begegnet wird, zeigt sich in einer der Elbischen Sagen, in der die Reintaler Burschen einen gegen das Gelübde der Keuschheit verstoßenden Priester mit dem Tod bestrafen (ebd.: 376) und - nicht zuletzt auch im Anspruch auf die (Re)Sozialisierung bzw. gesellschaftliche Reintegration in den Gemeinschafstraum. Charakteristisch für diesen ist die Verkoppelung des Rationalismus mit der Religion, verkörpert durch um die Aufklärung des Volkes bemühte Geistliche - jene zwischen dem Inneren des genannten Studierzimmers und dem Äußeren hin und herwechselnde Antipoden zum lüsternen Pfarrer aus Reintal - die als Referenz für vernunftmäßiges Verhalten vor Augen geführt werden.

Das sagenhafte Gottscheerland erscheint als ein nach außen und innen offener Kommunikationsraum mit prädominierender Naturprägung und mythischer sowie religiöser Kodierung. Die letzte kommt besonders in Gründungssagen zum Vorschein. Der Kommunikationsraum wird besiedelt von den durch Erfahrung der Transgression geographisch, ethnisch, sozial, kulturell und geschlechtlich kodierter Räume gekennzeichneten Einzelnen: Die Wirkung der genannten magischen Salben zum Sich-Verhexen erstreckt sich emanzipierend auf beide Geschlechter (ebd.: 400). Die Vielfalt räumlicher Transgressionen liefert notwendige Voraussetzungen für Individuierung, was besonders an Hexensagen zu beobachten ist, die schließlich in die angestrebte Resozialisierung mündet. Transgressionen ethnischer und sozio-kultureller Räume erfahren einige Ausnahmen und zeigen Risse in Geschichts- und Gründungssagen. Darin werden die Franzosen als die von der Zähigkeit der Gottscheer tief beindruckten und daher auf ihre Rachepläne verzichtenden Eindringlinge (ebd.: 427) und die Türken als von der Magie des Gottscheerlandes bezwungene (ebd.: 420) oder dank der heimatlichen Magie überlistete Räuber, Plünderer und Mörder vor Augen geführt. Feste ethnische und kulturelle Grenzen werden im Repräsentationsraum der religiösen Gründungssagen gezogen in der Beziehung zu dem verfremdeten Anderen, den Slowenen. Das Konkurrenzverhältnis zwischen beiden Gesellschaftsgruppen wird überblendet durch die Inszenierung der Gottscheer als moralisch und mentalitätsmäßig Überlegene:

Der Bau der Kirche wurde wieder aufgenommen und mit Hilfe der umwohnenden Deutschen glücklich vollendet. Die benachbarten Slawen versagten dabei jede Hilfe. Auch zum Ankaufe der neuen Glocken wollten sie nichts beisteuern. (ebd.: 416)

\section{ABSCHLUSSÜBERLEGUNGEN}

Den Volkssagen der Gottscheer sind beide Konzepte des Raumes eingeschrieben. Prädominierend ist zwar das Behälter-Konzept, das den Raum als Heimat ausweist. Zugleich jedoch wird dieses Raumkonzept subversiv unterlaufen durch rationalistische kritische Stimmen sowie gesellschaftlich transgredierende Verhaltenspraxen, die Raum 
als eine auf Veränderbarkeit deutende Dynamik sozialer Beziehungen und darüber hinaus innenkulturelle Differenz bzw. „Lücken und Lügen“ des Selbst in Erscheinung treten lassen.

Zwecks kultureller Homogenisierung und kollektiver Vergemeinschaftung wird der Repräsentationsraum der Sagen eingebettet in das Regionale - mit dem Verweis auf das Sagengut der Kärntner und als repräsentierter Raum, mit Koordinaten, die im Süden nach Kroatien, d. h. jenseits der Kulpa, nach Delnice, zu dem genannten Berg Klek, dem ,gottscheabarischen“ und kroatischen Brocken, und nach Rijeka sowie im Norden bis nach Laibach und Kärnten reichen. Durch das stellenweise Aufbrechen des mit Heimatlichen verknüpften Regionalen - symbolisch herbeigeführt nicht zuletzt auch durch die Einbindung von dem den Grimmschen Deutschen Sagen entnommenen Saal der Ahnen - wird das Sagengut der Gottscheer als Bestandteil der deutschen Volkskultur im Repräsentationsraum eines Nationalkonzepts positioniert. Dessen Anspruch auf universale Geltung zerbricht an der im Konstrukt sittlicher Überlegenheit des Eigenen verorteten Abgrenzung von ethnisch kodierter Alterität.

\section{Literatur}

BAUSINGER, Hermann (1980) Formen der „Volkspoesie“. 2. verbesserte und vermehrte Auflage. Berlin: E. Schmidt.

BREDHNICH, Rolf Wilhelm (1996) „Vorwort.“ In: R. W. Bredhnich/W. Suppan (Hrsg.), Gottscheer Volkslieder. Volksbaladen. Bd. 1. Mainz: B. Schott's Söhne, 9-91.

BREGER, Claudia/Tobias DÖRING (1998) „Einleitung: Figuren der/des Dritten.“ In: C. Breger/T. Döring (Hrsg.), Figuren der/des Dritten. Erkundungen kultureller Zwischenräume. Amsterdam/Atlanta: Rodopi, 1-18.

FERENC, Mitja (1993) Gottschee. Das verlorene Kulturerbe der Gottscheer Deutschen. Izgubljena kulturna dediščina kočevskih Nemcev. Ljubljana: Muzej novejše zgodovine.

„Gottscheer Persönlichkeiten.“ Gottscheer Zeitung. Mai 2005. http://wwwu.uni-klu. ac.at/hleustik/gottschee/leute/pdf/w-tschinkel01.pdf (16.05.2020).

HAUFFEN, Adolf (1895): Die deutsche Sprachinsel Gottschee. Geschichte und Mundart, Lebensverhältnisse, Sitten und Gebräuche, Sagen, Märchen und Lieder. Graz: Universitäts-Buchdruckerei und Verlags-Buchhandlung Styria.

HERDER, Johann Gottfried (1889 [1777]) „Vorrede der Volkslieder.“ In: J. G. Herder, Stimmen der Völker in Liedern. Halle: Otto Hendel, 61-78.

KREVS BIRK, Uršula (2019) „Zu einigen Aspekten des Deutschen als Kontaktsprache des Slowenischen." Linguistica 59/1, 155-173.

LEFEBVRE, Henri (2006) „Die Produktion des Raums.“ In: J. Dünne/S. Günzel (Hrsg.), Raumtheorie. Grundlagentexte aus Philosophie und Kulturwissenschaften. Frankfurt a. Main: Suhrkamp, 330-342.

MÜLLER, Jakob (2014) Kočevar ima samo eno domovino. Göttscheabar hot lai oin Hoimöt. Grosuplje: Kulturno društvo sv. Mihaela. 
PETZOLD, Leander (1999) Einführung in die Sagenforchung. Konstanz: UVK-Universitätsverlag.

REINHARDT, Udo (2012) Mythen - Sagen - Märchen. Eine Einführung mit exemplarischen Motivreihen. Freiburg im Breisgau/Berlin/Wien: Rombach Verlag.

SOJA, Edward W. (2005) „Die Trialektik der Räumlichkeit.“ In: R. Stockhammer (Hrsg.), TopoGraphien der Moderne. Medien zur Repräsentation und Konstruktion von Räumen. München: Wilhelm Fink, 93-123.

TSCHINKEL, Wilhelm (2004): Kočevarska folklora v šegah, navadah, pravljicah, povedkah, legendah in drugih folklornih izročilih. Gottscheer Volkstum in Sitte, Brauch, Märchen, Sagen, Legenden und anderen volkstümlichen Überlieferungen. Hrsg. von A. P. Florjančič/M. Stanonik. Ljubljana: Založba ZRC.

„Tschinkel, Wilhelm(1875-1938), Lehrer und Volkskundler.“ Österreichisches Biographisches Lexikon. https://www.biographien.ac.at/oebl?frames=yes. (16.04.2020).

WOLFRAM, Richard (1980) Brauchtum und Volksglaube in der Gottschee. Wien: Selbstverlag des Österreichischen Museums für Volkskunde.

\section{Zusammenfassung \\ VOLKSSAGEN DER GOTTSCHEER: ASPEKTE DES RAUMES}

Die Sagen der Gottscheer, enthalten in dem für die Sammlung und den Transfer ihrer Volksdichtung grundlegenden, von dem Gottscheer Wilhelm Tschinkel veröffentlichten Werk Gottscheer Volkstum in Sitte, Brauch, Märchen, Sagen, Legenden und anderen volkstümlichen Überlieferungen (1932), weisen wegen des inselhaften Charakters des Gottscheer Sprachgebietes außerordentliche stoffliche und thematische Urwüchsigkeit auf.

Bei der ästhetischen Verräumlichung kommt in den Sagen neben Pflanzen und Gegenständen besondere Bedeutung den magischen Handlungen zu, die an karsttypische, auf die Vergangenheit bezogene Naturräume gebunden sind. Die Raumpraxis besteht aus einer Kette von Stimmung erzeugenden Handlungsrekurrenzen, die von Herausforderung bis Bezwingung des Übernatürlichen reichen, für eine Reihe von Verrückungen in den Beziehungen zwischen dem Unheimlichen und dem Vertrauten am angestammten Ort sorgen und die Unergründbarkeit des menschlichen Lebens deutlich in Erscheinung treten lassen. Die innere Raumdialektik lässt die Gottscheer als von vormodernen und modernen Verhaltens- und Handelspraxen geprägte, in einem nach Innen und Außen hin offenen Kommunikationsraum verortete und durch zahlreiche Transgressionen von geographischen, ethnischen, sozialen, kulturellen und geschlechtlich kodierten Räumen gekennzeichnete Individuen erscheinen. Während ihre als Bestandteil der deutschen Volkskultur repräsentierten Sagen sie im Repräsentationsraum eines universale Geltung beanspruchenden Nationalkonzepts positionieren, erfahren Raumtransgressionen durch sittliche Abgrenzungen in der Beziehung zu den Eindringlingen, wie Franzosen und Türken, und nicht zuletzt in ihrer Eigenschaft der verfremdeten Andersheit auch zu den Slowenen sichtbare Risse. 
Schlüsselwörter: Gottscheer Volksagen, Wilhelm Tschinkel, übernatürliche Raumpraxis, Repräsentation von karsttypischen Naturräumen, Raumtransgression und Raumfixierung

\section{Abstract \\ GOTTSCHEER FOLK MYTHS: ASPECTS OF SPACE}

The myths of the Gottscheer Germans, included in Gottscheer Volkstum in Sitte, Brauch, Märchen, Sagen, Legenden und anderen volkstümlichen Überlieferungen, a work of central importance for the collection and transmission of Gottscheer folk poetry published in 1932 by Wilhelm Tschinkel, a Gottscheer himself, show extraordinary primitiveness of the literary material and topics, which reflects the nature of the Gottschee area as a linguistic and cultural enclave. In terms of aesthetic spatialization, magical actions, objects and plants play a central role in Gottscheer myths, and are associated with the natural environment typical of the Karst region. The spatial practice of referring to the past engenders mood creating recurrent actions, ranging from challenging the supernatural to conquering it. They result in shifting borders between the frightening and the familiar on ancestral soil and reveal the unfathomable nature of human life. From the perspective of the internal spatial dialectic, the Gottscheer appear as individuals characterized by pre-modern as well as modern practices of behaviour and action, located within a dynamic communicative space and marked by multiple transgression of geographically, socially, culturally and gender encoded spaces. While the myths, represented as a component of German folk poetry, place the Gottscheer within both a universal and national representational space, the moral spatial transgressions related to intruders such as the French and Turks, but also Slovenes, unambiguously point to cracks triggered by a foreign Otherness.

Keywords: Gottscheer folk myths, Wilhelm Tschinkel, supernatural spatial practices, representation of natural spaces typical of the Karst, spatial transgression and fixation

\section{Povzetek \\ KOČEVARSKE LJUDSKE BAJKE - PROSTORSKI VIDIKI}

Bajke iz zbirke Gottscheer Volkstum in Sitte, Brauch, Märchen, Sagen, Legenden und anderen volkstümlichen Überlieferungen (1932) Wilhelma Tschinkla, dela, ki je za zbiranje in transfer kočevarske ljudske kulture izrednega pomena, odlikuje izjemna snovna in tematska prvobitnost. Za estetsko poprostorjenje so $\mathrm{v}$ bajkah poleg magičnih predmetov in rastlin osrednjega pomena ravnanja in dejanja magičnega značaja, vezana na preteklost in na prostore kraške naravne krajine. V preteklost umeščeno prostorsko prakso sestavlja niz ponovnih dogajalnih pojavitev oz. dogajalnih rekurenc, ki ustvarjajo razpoloženja. Dogajalne rekurence segajo od izzivanja do obvladovanja 
nadnaravnega in sprožajo vrsto premikov med srh zbujajočim skrivnostnim in poznanim na domačih tleh ter pričajo o nedojemljivosti človeškega življenja. V luči notranje dialektike prostora se Kočevarji kažejo kot v dinamični komunikacijski prostor umeščeni posamezniki, ki jih opredeljujejo moderne in predmoderne prakse ravnanja in delovanja ter številne transgresije geografskih, etnično, socialno, kulturno in spolno kodiranih prostorov. $Z$ bajkami, predstavljenimi kot del nemške ljudske kulture, se Kočevarji umeščajo $\mathrm{v}$ reprezentacijski prostor nacionalnega, z univerzalno intenco. Hkrati pa se v prostorskih transgresijah preko moralnih razmejitev v odnosu do vsiljivcev, kot so v bajkah Francozi in Turki, in nenazadnje tudi do Slovencev kot potujene drugosti pokažejo očitne razpoke.

Ključne besede: Kočevarske ljudske bajke, Wilhelm Tschinkel, nadnaravna prostorska praksa, reprezentacija kraških naravnih prostorov, transgresija in fiksiranje prostora 